Research Paper

\title{
Data-based Modification System of LeanMAP Contents for Automated Driving
}

\author{
Takuma Ito $^{\text {1) }}$ Satoshi Nakamura $^{1)}$ Kyoichi Tohriyama ${ }^{2)}$ Minoru Kamata $^{1)}$ \\ 1) The University of Tokyo \\ 5-1-5 Kashiwanoha, Kashiwa, Chiba, 277-8589, Japan (E-mail: ito@iog.u-tokyo.ac.jp) \\ 2) Toyota Motor Corporation \\ 1200 Mishuku, Susono, Shizuoka, 410-1193, Japan
}

Received on December 15, 2017

\begin{abstract}
To realize a digital map for automated driving on non-highway roads, where plans for preparing highprecision 3D maps in the near future have not been determined, we propose "LeanMAP", the contents of which are composed of map data from car navigation systems. In addition, to improve the precision of the LeanMAP contents, we propose a modification system based on actual driving data. Through the results of pilot trials on data modification in left turns at an intersection and gentle curves at a non-intersection part of a public road, we confirm the feasibility of our proposed system in that it correctly modifies the contents of LeanMAP.
\end{abstract}

KEY WORDS: Safety, Intelligent vehicle, Driving support / Digital map, LeanMAP, Actual driving data [C1]

\section{Introduction}

Thus far, various technologies have been developed for automated driving; furthermore, technology regarding digital maps $^{(1)}$ is one of the most essential components for the realization of automated driving on actual public roads. Digital maps are expected to be used for various purposes, such as path planning, localization, and long-range sensing. To date, two major approaches of digital maps for automated driving have been proposed: digital maps that are combined with data from the Real Time Kinematic Global Positioning System (RTK-GPS) ${ }^{(2)}$, and the Simultaneous Localization and Mapping (SLAM) method with all-around multilayer Light Detection and Ranging $(\text { LIDAR })^{(3)}$. Although automated driving has been realized via both methods at a research level, there are still certain problems such as the cost of special sensors and the vast amount of map data for practical realization of automated driving. Thus, in our previous study, we proposed a "LeanMAP concept" (4) and developed sensing systems based on LeanMAP. Furthermore, we developed the LeanMAP framework in which the digital map contents and the sensing system act jointly in a complementary manner. Moreover, we confirmed their feasibility through experiments on automated driving using a test track. However, as we aimed to confirm the feasibility of the concept in our previous study, we prepared the contents of LeanMAP by manually reducing the precise $3 \mathrm{D}$ digital map. However, regarding nonhighway roads, as precise 3D digital maps are not planned to be created in the near future, we need to develop a method that does not require precise 3D digital maps. Therefore, we focused on the digital maps used in car navigation systems, which have already been maintained for most roads in Japan. Fig. 1 shows the conceptual schematic of the preparation of the LeanMAP contents. Because digital maps for car navigation systems have originally been designed for route navigation and information support, their precision is not sufficient for automated driving. Thus, we need to discuss two topics: the concept of a preliminary method for converting the digital map contents of car navigation systems to LeanMAP contents, and the concrete modification method of the LeanMAP contents. From these perspectives, the purpose of this study is to confirm the feasibility of the LeanMAP based on the contents of the car navigation system through prototype development and the initial evaluation on a public road.

\begin{tabular}{|c|c|c|c|c|c|}
\hline \multicolumn{2}{|c|}{$\begin{array}{l}\text { Precise 3D } \\
\text { digital map }\end{array}$} & $\begin{array}{l}\text { Previous } \\
\text { approach }\end{array}$ & & $\begin{array}{l}\text { Proposed approach } \\
\text { in this study } \\
\end{array}$ & $\begin{array}{c}\text { Car navigation } \\
\text { map }\end{array}$ \\
\hline & \multicolumn{2}{|c|}{ Precise 3D digital map } & "LeanMAP" & \multicolumn{2}{|c|}{ Car navigation map } \\
\hline Purpose & \multicolumn{2}{|c|}{$\begin{array}{c}\text { Localization and } \\
\text { vehicle control }\end{array}$} & $\begin{array}{l}\text { Localization and } \\
\text { vehicle control }\end{array}$ & \multicolumn{2}{|c|}{$\begin{array}{c}\text { Navigation and } \\
\text { information provision }\end{array}$} \\
\hline Target road & \multicolumn{2}{|c|}{$\begin{array}{c}\text { Highway and } \\
\text { limited number } \\
\text { of national roads }\end{array}$} & Almost all roads & & All roads \\
\hline Accuracy & \multicolumn{2}{|c|}{ Less than $0.1 \mathrm{~m}$} & $0.5 \mathrm{~m}$ (As a requirement) & & $5 \mathrm{~m}$ or more \\
\hline $\begin{array}{c}\text { Amount of } \\
\text { data }\end{array}$ & \multicolumn{2}{|c|}{$\begin{array}{l}\text { Too vast to be mounted } \\
\text { on vehicles }\end{array}$} & $\begin{array}{l}\text { Desired to be small enough to } \\
\text { be mounted on vehicles }\end{array}$ & Small er & $\begin{array}{l}\text { enough to be mounted } \\
\text { on vehicles }\end{array}$ \\
\hline
\end{tabular}

Fig. 1 Conceptual Schematic of the LeanMAP Preparation

\section{LeanMAP Framework for Automated Driving}

\subsection{Concept of the LeanMAP framework}

As discussed in Chapter 1, we previously developed the LeanMAP framework, which consisted of recognition systems that were combined with the LeanMAP contents in a complementary manner to address the existing problem pertaining to the vast amount of precise 3D map data. Fig. 2 shows the conceptual schematic of the LeanMAP framework. 


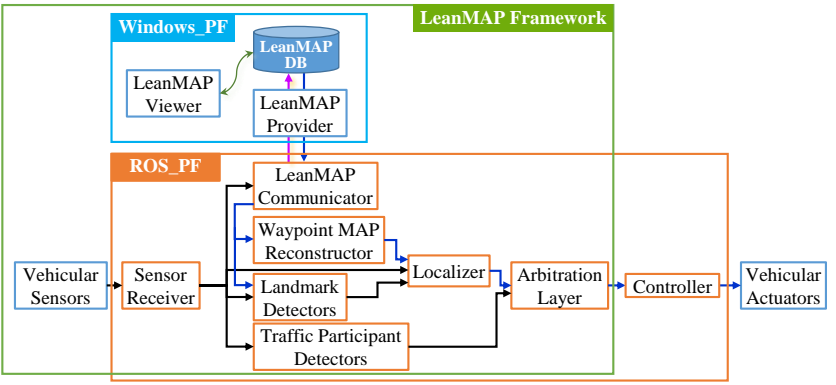

Fig. 2 Conceptual Schematic of the LeanMAP Framework

In our previous study, we selected the necessary elements of the traffic environment to dynamically reconstruct the waypoint map for vehicle control and the landmark map for localization. Then, we manually prepared the LeanMAP contents that pertained to the waypoint map and the landmark map from the data of the precise 3D digital map. The following is the necessary information for each map.

- Waypoint map: Information regarding the curve segment.

- Landmark map: Information regarding the characteristics of the landmark, such as stop lines, crosswalks, and speed limit signs.

The position information of the abovementioned elements is expressed via the following basic information. Fig. 3 shows the corresponding conceptual schematic of the LeanMAP data structure.

- Node: Intersection that can be searched by the system.

- Link: Directed route between intersections.

- Lane: Driving course including an intersection at a certain link. There are one or more lanes in a link.

- Offset: Distance projected to the lane from the origin point of the lane to various points, such as the current position of the vehicle and the position of landmarks.

In the existing research works, typical systems with precise 3D digital maps preliminarily prepared the vast amount of information regarding the elements of traffic environments based on global coordination systems. On the other hand, in our study, the LeanMAP system encompasses a simple model for the intersection and uses the concept of offset, which is the projected driving distance from the origin point of the lane, as shown in Fig. 3. Moreover, a characteristic of the LeanMAP system is that it uses various pieces of information while dynamically reconstructing the elements of the traffic environments along the current lane based on the local coordination system, which, in its turn, is based on the origin point of the lane.

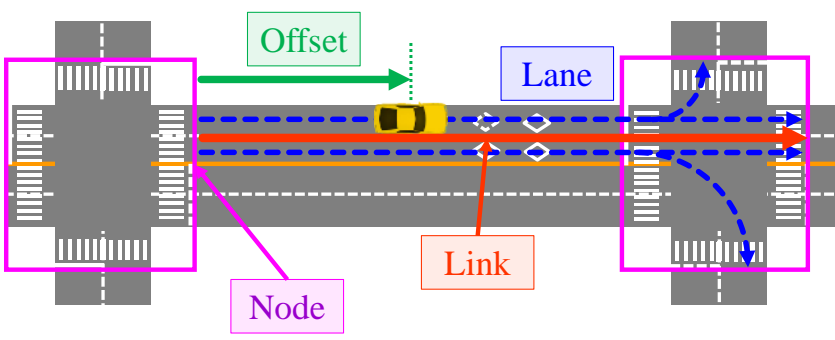

Fig. 3 Conceptual Schematic of the LeanMAP Data Structure

Regarding the recognition systems shown in Fig. 2, first, sensor receivers communicate with various sensors and provide the sensor information to the functions that follow in succession. Second, the LeanMAP communicator provides the GPS information to the LeanMAP provider. Third, the LeanMAP provider roughly estimates the current position based on the GPS information, and provides various types of information about nearby elements of the traffic environments such as landmarks and curves. Fourth, the waypoint map reconstructor dynamically reconstructs the waypoints using the information of the curve, which consists of three pieces of information: the "offset," "R," and " $\theta$." Fig. 4 shows the schematic of reconstructing a waypoint map. The offset denotes the position of start point of the curve, $R$ denotes the radius of the curve, and $\theta$ denotes the angle of the curve. It is characteristic that a LeanMAP system can reconstruct the waypoint map by using only the information of the curve segments. Fifth, landmark detectors detect the surrounding landmarks based on the information from the LeanMAP provider, and calculate the precise longitudinal position by comparing the estimated relative distance to the detected landmark with the LeanMAP contents that indicate the offset of the landmark.

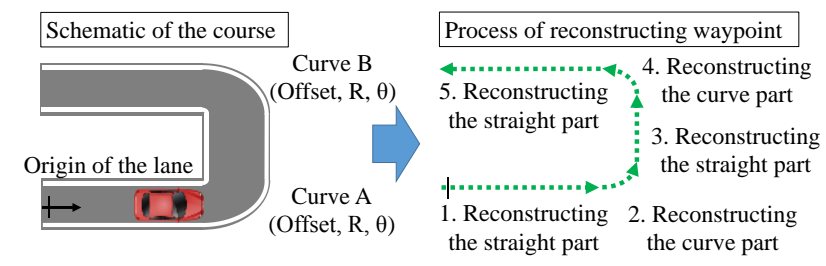

Fig. 4 Schematic of Reconstructing a Waypoint MAP

The LeanMAP framework performs localization on the reconstructed waypoint map using the longitudinal position, which is based on the landmark detection, and the lateral position, which is based on the lane mark detection. Thus, the current position of the vehicle in the LeanMAP framework is expressed through the offset of the nearest waypoint and the deviation from the nearest waypoint. The deviation consists of the lateral deviation (LD) and the deviation angle, $\phi$. The position of the nearest waypoint is expressed as $\mathrm{x}, \mathrm{y}, \theta$, and offset. Fig. 5 shows the schematic of the waypoint coordinate system. In this study, the resolution of the waypoint was $0.05 \mathrm{~m}$. Based on the current position and the information from the forward waypoint map, the controller calculates the control values while considering the surrounding traffic participants that are detected by the vehicular sensors.

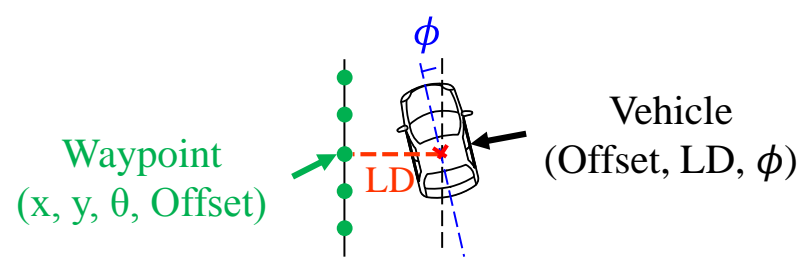

Fig. 5 Schematic of the Waypoint Coordinate System

2.2. Introducing the Map Data of the Car Navigation System into the LeanMAP Framework

In our previous study, we aimed to confirm the feasibility of the LeanMAP framework; hence, we prepared the data of the LeanMAP contents by manually extracting necessary information 
from the precise 3D digital map. However, regarding the practical use of the LeanMAP framework in various roads in Japan, two problems still remain unresolved. First, although a precise 3D digital map for the highway is planned to be created by 2020 , there are no specific plans for preparing a precise 3D digital map for the non-highway roads, particularly in the country area. Second, as current methods for the preparation of digital maps are not well systemized, we cannot apply the current method to prepare the nationwide data of the LeanMAP contents of nonhighway roads.

To solve these problems, we focused on the digital map of car navigation systems. The merits of using the digital map of car navigation systems are the following.

- Certain parts of the digital map contents of car navigation systems for $74 \%$ of all roads in Japan have already been maintained; hence, the initial introduction is relatively easy. In addition, the methods for maintaining the digital maps of car navigation systems have already been systemized.

- The "Node" and the "Link," which comprise the basic information about the intersection that has been defined in the LeanMAP, are based on the architecture of the road network defined by the Digital Road Map (DRM) ${ }^{(5)}$, which is used for the contents of car navigation systems as well; therefore, we can easily prepare the contents of LeanMAP from the contents of car navigation systems.

2.3. The Problem of LeanMAP Based on the Map Data of the Car Navigation System

The digital map of car navigation systems aims to navigate the drivers; hence, a variety of information may not be sufficient for the precise control of the vehicle. Thus, when we prepare the LeanMAP contents that the digital map of the car navigation system does not include in its database, we need to assign temporary values. To be more precise, we assigned temporary values of curve information for the reconstruction of a waypoint map, and temporary values of landmark information for localization. The LeanMAP with temporary contents will hereafter be referred to as "LeanMAP Float."

Figure 6 shows the conceptual schematic of the intersection curve in the LeanMAP Float. In the LeanMAP framework, the intersection curve presents three types of information, namely the offset, $\mathrm{R}$, and $\theta$. Among these values, as $\theta$ can be calculated from the original contents of the digital map of the car navigation system, the precision of $\theta$ is relatively satisfactory. On the contrary, as the contents of the digital map of the car navigation system do not include information regarding the offset and R, we need to assign temporary values to these variables. More specifically, we assigned $\mathrm{R}$ to be equal to two times the value of the width of the original lane, and the offset of the intersection curve to be equal to the offset of the stop line.

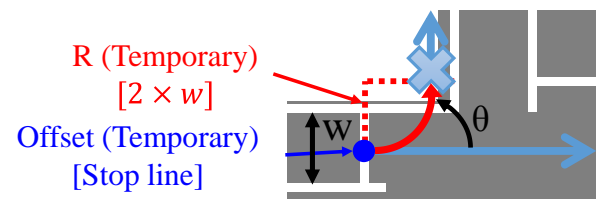

Fig. 6 Schematic of the Intersection Curve in LeanMAP Float

Figure 7 shows the conceptual schematic of the nonintersection curve in LeanMAP Float. As shown in Fig. 7, the non-intersection curve consists of small arc segments, which are based on certain curve composition points, and of interpolated straight segments. The non-intersection curve contains the same type of information as the intersection curve. Regarding the offset values of the curve composition points, we assumed that their precision was relatively high. In contrast, as the contents of the digital map of a car navigation system do not include information regarding $\mathrm{R}$ and $\theta$, we assigned temporary values. Regarding $\mathrm{R}$, we assigned the value of $10.0 \mathrm{~m}$ for all points. Regarding $\theta$, we calculated the temporary values from the database of the car navigation system.

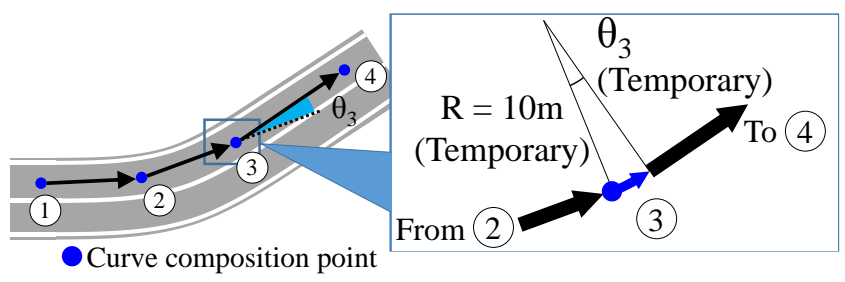

Fig. 7 Schematic of the Non-Intersection Curve in LeanMAP Float

Regarding the abovementioned temporary values, if we use high-specification sensors, we can realize automated driving even when the LeanMAP Float is based on temporary values. However, such request for excessively high-specification sensors creates cost issues in practical use; therefore, we need to balance the specifications of the vehicular sensors and digital maps. Based on these discussions, we focused on the modification system of the LeanMAP contents to improve the precision of the LeanMAP Float. We will refer to the LeanMAP with improved contents as "LeanMAP Fix." Fig. 8 shows the conceptual process of generating the LeanMAP Fix from the car navigation map. Here, the important point is that because the LeanMAP Fix uses vehicular sensors to a certain extent, the LeanMAP Fix does not require the excessively high levels of precision that precise 3D digital maps have. In other words, the required precision of the LeanMAP Fix is practical when vehicular sensors are used together with the LeanMAP framework in a complementary manner. 


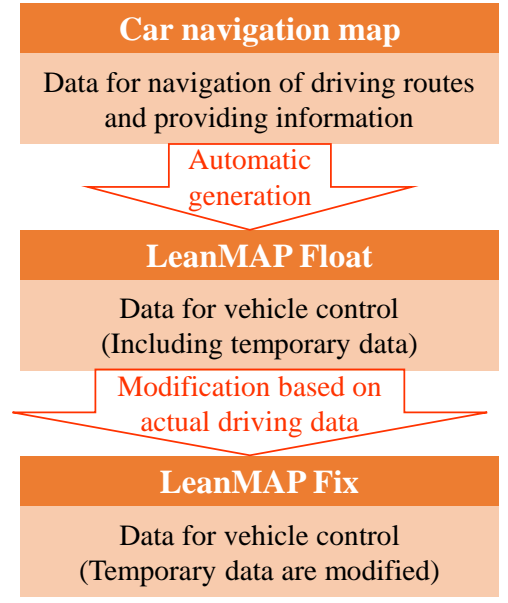

Fig. 8 Process of Generating the LeanMAP Fix from the Car Navigation Map

\section{Modification System of the LeanMAP Contents by Referring to Actual Driving Data}

\subsection{Concept Design of the Modification System}

To develop the modification system of the LeanMAP contents - which consists of temporary values, as discussed in Section 2.3-we focused on the trajectories that had been calculated with the wheel velocity sensor and the inertial measurement unit (IMU). The information regarding the curve segment and the landmarks is different for each lane, and the information obtained in each lane is independent from that obtained in other lanes; therefore, we could modify the LeanMAP contents without considering their consistency with the contents of other lanes. An important point in the modification is the sequence in which each of the contents within a certain lane is modified; hence, we need to discuss the constraints between each of the contents.

A vehicle with LeanMAP frameworks cannot localize in the intersection segment; therefore, the modification of the contents pertaining to the intersection curve is of the highest importance. The contents of the landmarks are second in priority because the time-series vehicle offset from the tip of the lane to the end of the lane is correctly calculated using the modified contents of the landmarks. Next, we need to modify the contents of the nonintersection curves.

Figure 9 shows the update flow of the LeanMAP modification system. First, we collect the driving data that include the driving on the target lane. Then we remove invalid data, such as trips in which the vehicle changes lanes owing to a parked vehicle and trips where the vehicular sensors output abnormal values, by checking the sensor data and the detection results. Next, we find the tip and the end of the lane by checking the detection results for the crosswalks and stop lines, and we extract the segment of analysis for each data set. Finally, we modify the contents of LeanMAP from the extracted driving data. The detailed process of modification will be explained in the following sections.

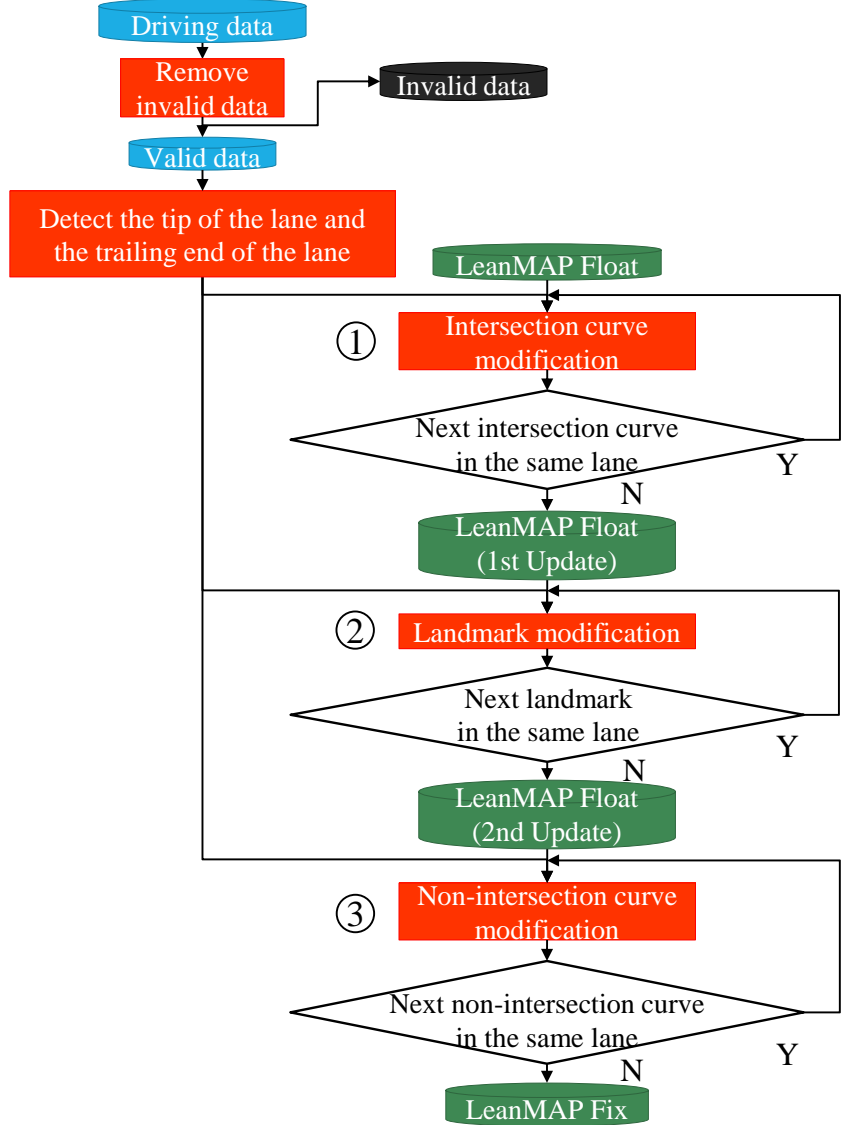

Fig. 9 Update Flow of the LeanMAP Modification System

In the proposed system, we calculated the lateral deviations and the angle deviation between the vehicle posture and the lane using the camera-based detection results of the lane marks. From these deviations and vehicle trajectories, we estimated the center of a lane in order to exclude the effect of driving styles, such as driving on the left side of the lane. Fig. 10 shows the conceptual schematic of the center estimation of a lane.

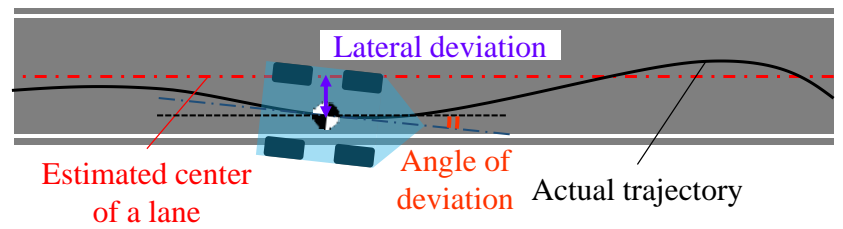

Fig. 10 Conceptual Schematic of the Estimated Center of a Lane

\subsection{Modification of the Intersection Curve Data}

Figure 11 shows the conceptual schematic of the modification of the intersection curve data. As discussed in Section 2.3, the LeanMAP contents of the intersection curve encompass three types of information: the offset, $\mathrm{R}$, and $\theta$. First, we estimate $R$ and $\theta$ from the combination of the time-series velocity and the yaw rate of the vehicle. Then, we optimize the offset value of the curve by comparing the trajectories with the reconstructed waypoint. 


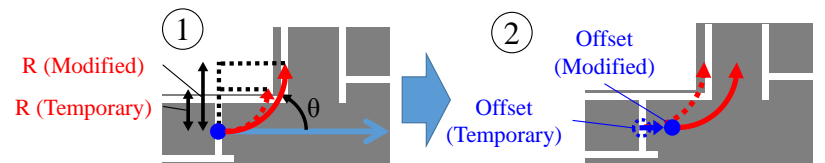

Fig. 11 Conceptual Schematic of the Modification of the Intersection Curve Data

\subsection{Modification of Landmark Data}

Figure 12 shows the conceptual schematic of the modification of the landmark offset. Regarding the LeanMAP contents of landmarks, the type information of landmark is correct because the original contents of the digital map of the car navigation system contain this type of information. Thus, we need to modify their offset information. Using the actual driving distance, the detection result of the tip of the lane, and the detection result of the target landmark, our system calculates the actual offset values of the landmark. In addition, we modified the offset values of other LeanMAP contents, such as the curve information that exists between the tip of the lane and the modified landmark by referring to the modified offset of the landmark.

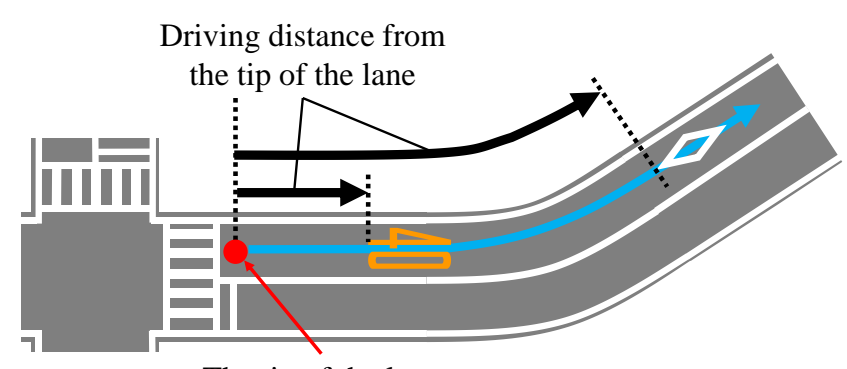

The tip of the lane

Fig. 12 Conceptual Schematic of the Modification of Landmark Data

\subsection{Modification of the Non-Intersection Curve Data}

Figure 13 shows the conceptual schematic of the modification of the non-intersection curve data. Regarding the non-intersection curve, we need to modify the $\mathrm{R}$ and $\theta$ of each curve composition point. First, we estimate the average $\mathrm{R}$ around each point from the combination of the time-series velocity and yaw rate. Then, we calculate the average $\theta$ around each point. This average $\theta$ is an initial candidate value for the modified $\theta$. Next, we optimize $\theta$ by referring to the preview gap between the next curve composition point and the corresponding point of the trajectory. We repeat this sequence of procedures starting from the first curve composition point. Regarding the final curve composition point, as the waypoint does not include any more points, we define an imaginary point that is only used for the optimization of the final point. We place an imaginary point ahead of the final point; the distance between them is equal to the distance between the final point and the point immediately before the final point. If the intersection curve point is closer to the final point compared with the imaginary point, we use this point instead of the imaginary point.

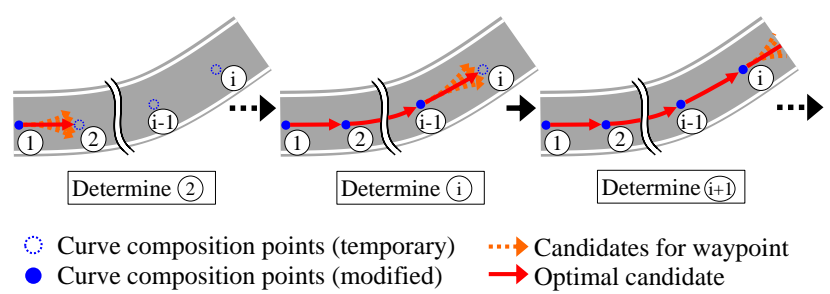

Fig. 13 Conceptual Schematic of the Modification of the Non-Intersection Curve Data

\section{Feasibility Test Based on Actual Driving Data}

\subsection{Abstract of the Feasibility Test}

To confirm the feasibility of the modification system of LeanMAP, we conducted an evaluation test on a public road. Because the main topic in this study is the localization with LeanMAP, we collected the driving data via manual driving, and not via automated driving. In this test, we used following vehicular sensors for each of the corresponding functions.

- IMU: Estimation of vehicle motion;

- Monocular camera: Detection of stop lines;

- Monocular camera: Detection of the lane mark;

- Wheel velocity sensor: Estimation of the driving distance.

Figure 14 shows the schematic of the course for the feasibility test. After driving past the $\mathrm{T}$ junction, there are rightward non-intersection curves. At the end of the course, the vehicle turns left at the intersection. In this course, we collected the driving data of 30 trips in total. Because there were 3 trips containing abnormal data, we used the data of the remaining 27 trips for the modification of the LeanMAP contents. For almost all areas along the course, the vehicle could correctly detect the lane mark. In this course, there was only one landmark for localization, namely the stop line immediately prior to the intersection.

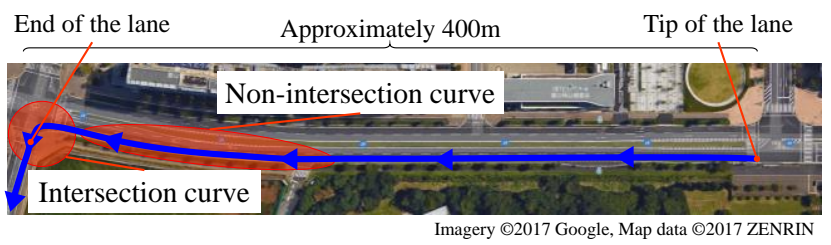

Fig. 14 Schematic of the Course for the Feasibility Test

\subsection{Modification Result of the Intersection Curve Data}

To modify the LeanMAP contents of the intersection curve, we executed the following three steps.

1. Modification of $\mathrm{R}$ and $\theta$,

2. Translation and rotation of trajectories to superimpose them on the reconstructed waypoint,

3. Modification of the offset of the curve.

The first process was the modification of $\mathrm{R}$ and $\theta$. First, we determined the start point and the end point of the curve segment based on the estimated time-series curvature, which can be calculated using the time-series yaw rate and wheel velocity. The curvature can be calculated using the following equation.

$$
1 / \mathrm{R}=\gamma / \mathrm{V}
$$


where $\mathrm{R}$ denotes the curvature radius, $\gamma$ denotes the yaw rate, and $\mathrm{V}$ denotes the vehicle velocity. Fig. 15 shows an example of the calculated curvature at the intersection, based on a certain trip. As shown in the figure, we set the first point where the curvature became more than $0.01 \mathrm{~m}^{-1}$ as the start point of the curve segment. In a similar manner, we set the last point where the curvature became more than $0.01 \mathrm{~m}^{-1}$ as the end point. Based on the average deviation angle between the start point and the end point, we modified the $\theta$ of this curve. In addition, we divided the curve segment into three parts to eliminate the effect of the clothoid curve. Then, we calculated the average radius of the middle segment, and we set the value as the modified R. As a result of this process, $\theta$ was updated from the temporary value of 87 degrees, to the modified value of 88 degrees. In a similar manner, $\mathrm{R}$ was updated from the temporary value of $6.2 \mathrm{~m}$, to the modified value of $9.7 \mathrm{~m}$.

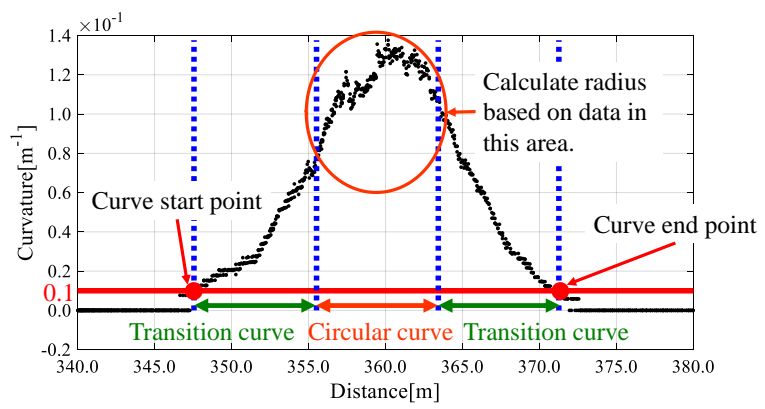

Fig. 15 Example of the Calculated Curvature at the Intersection Curve

The second process was the translation and rotation of the estimated lines of the lane center. The detailed process is as follows. Fig. 16 shows the result of this process.

1. We set the origin point on the reconstructed waypoint. The position of the origin point was $10 \mathrm{~m}$ before the stop line. In addition, we set the evaluation point for rotation, which was $10 \mathrm{~m}$ before the origin point.

2. For each trajectory, we estimated the corresponding origin point based on the detection result of the stop line. Next, we translated each trajectory in such a manner that the origin point of each trajectory would be located at the origin point of the reconstructed waypoint.

3. For each trajectory, we estimated the corresponding evaluation point for the rotation, based on the detection result of the stop line. Then, we rotated each trajectory in such a manner that the evaluation point for the rotation would be located as close to the evaluation point of the reconstructed waypoint as possible.

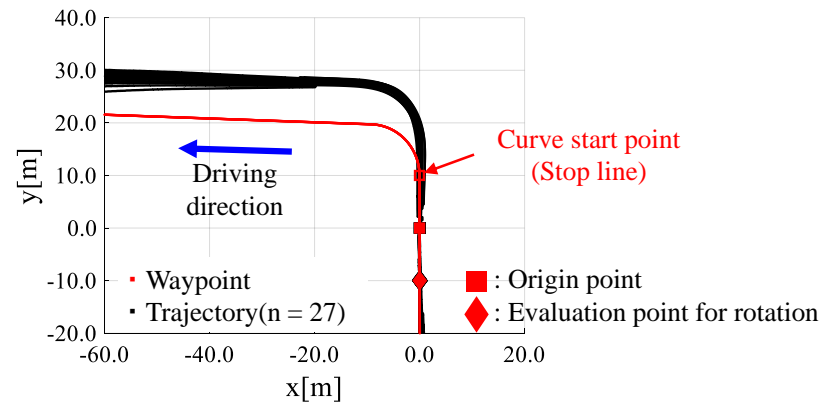

Fig. 16 Waypoint without Modification of the Curve Start Point and the Trajectories at the Intersection Curve

The third process was the modification of the offset of the curve. The detailed process is as follows. Fig. 17 shows the result of this process.

1. For the reconstructed waypoint, we set two evaluation points after the curve segment. The first evaluation point was located $20 \mathrm{~m}$ behind the end point of the curve. In a similar manner, the second evaluation point was located $30 \mathrm{~m}$ behind the end point.

2. For each trajectory, we set the corresponding evaluation points to be approximately $20 \mathrm{~m}$ and $30 \mathrm{~m}$ behind the end point of the curve.

3. We adjusted the curve start point and evaluated the deviation of the corresponding evaluation points between each trajectory and the reconstructed waypoint. Based on the minimum deviation conditions, we selected the optimized offset of the curve start point, and adopted the value as the modified offset of the curve.

As a result of this process, the offset was updated from the temporary value of $0.0 \mathrm{~m}$ to the modified value of $7.5 \mathrm{~m}$.

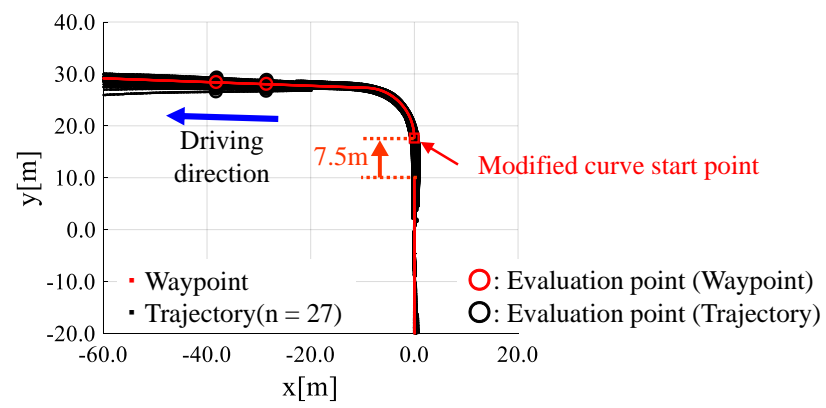

Fig. 17 Waypoint with Modification of the Curve Start Point and the Trajectories at the Intersection Curve

\subsection{Modification Result of Landmark Data}

In this course, the stop line in front of the intersection was the target of the modification. First, we calculated the distance from the tip of the lane to the stop line by referring to the driving distance and the detection result of the stop line for each of the driving data. Then, we calculated the average value of the distance and adopted the value as the modified offset of the stop line. As a result, the offset of the stop line was updated from the temporary value of $351.1 \mathrm{~m}$ to the modified value of $352.1 \mathrm{~m}$. In addition, we added the modified value, which was $+1.0 \mathrm{~m}$ in this 
case, to the offset value of the curve composition points that existed between the tip of the lane and the stop line.

\subsection{Modification Result of the Non-Intersection Curve Data}

In this course, there were five curve composition points, the contents of which required modification. For each trajectory, we selected the corresponding points for which the offset values were close to the offset values of each curve composition point. Then, we calculated the time-series curvature around the points of the trajectory that were located within $5 \mathrm{~m}$ from the corresponding points using Equation (1). In addition, we calculated the average value of the curvature radius using the inverse of the time-series curvature, and adopted the value as the initial $\mathrm{R}$ candidate for each curve composition point. Fig. 18 shows an example of the calculated curvature at the non-intersection curve. Next, we calculated the initial candidate of $\theta$ at each curve composition point using the deviation of the angles between two consecutive curve composition points.
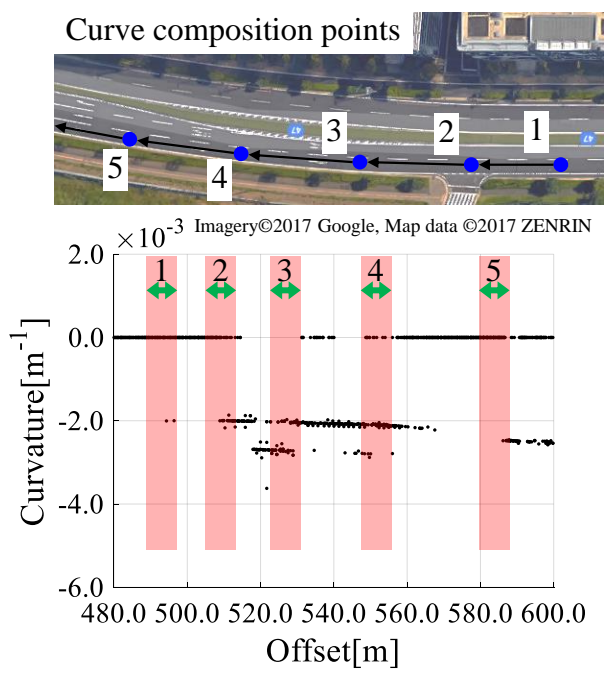

Fig. 18 Example of the Calculated Curvature at the Non-Intersection Curve

Based on the initial candidate values of $\mathrm{R}$ and $\theta$ of each curve composition point, we optimized $\mathrm{R}$ and $\theta$ by comparing each trajectory with the reconstructed waypoint map. The details of the comparison are as follows.

1. For the i-th curve composition point, we reconstructed the waypoints between the $\mathrm{i}$-th curve composition point and the next composition point based on the candidate values of $\mathrm{R}$ and $\theta$. Then, we optimized $\theta$ around the initial candidate values by minimizing the distance between the $(i+1)$-th curve composition point and the nearest point of each trajectory, as shown in Fig. 13.

2. For the i-th curve composition point, the length of the arc between the i-th point and the next point, which was derived by multiplying $\theta$ by $R$, should be smaller than the deviation value between the offset values of the $\mathrm{i}$-th point and the next point. However, the multiplication of the modified $\theta$ with candidate $\mathrm{R}$ may yield a higher value than the deviation value because the estimation would be rough. In such case, we adjusted the $R$ values to be equal to the deviation value divided by the modified $\theta$.

3. For all curve composition points, we repeated the abovementioned operation.

Fig. 19 shows the result from the update of the non-intersection curve data. For example, the value of $\theta$ of the 2nd curve composition points was updated from the temporary value of 2 degrees to the modified value of 5 degrees. In a similar manner, the value of $\mathrm{R}$ of the 2 nd curve composition point was updated from the temporary value of $10.0 \mathrm{~m}$ to the modified value of $237.2 \mathrm{~m}$

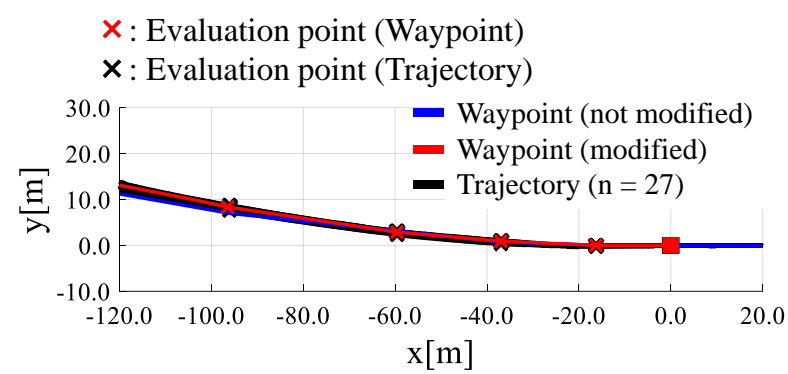

Fig. 19 Comparison between the Waypoint Map with Modification and the Waypoint Map without Modification

\subsection{Evaluation of the Adequateness of the Modified Waypoint}

To confirm the feasibility of the modification method of the LeanMAP contents, we evaluated the precision of the modified waypoint. In the LeanMAP framework, the complementary combination of the digital map with vehicular sensors contributes to the realization of automated driving; therefore, the improvement in the precision of the digital map minimizes the requirement for high-specification vehicular sensors. From this perspective, we evaluated the precision of the modified LeanMAP by temporarily ignoring the lane mark detection. Figs. 20 and 21 show the conceptual schematics of the evaluation at the intersection curve and non-intersection curve. First, we ignored the detection results of the lane mark at the evaluation area (these results had been useful in lateral localization), including those at the curve segment. Then, we performed the localization only by using the IMU and LeanMAP. After the evaluation area, we reactivated the lane mark detection. At this point, the correction value when the lane mark detection was re-activated indicated the precision of the waypoint map based on the LeanMAP contents. 


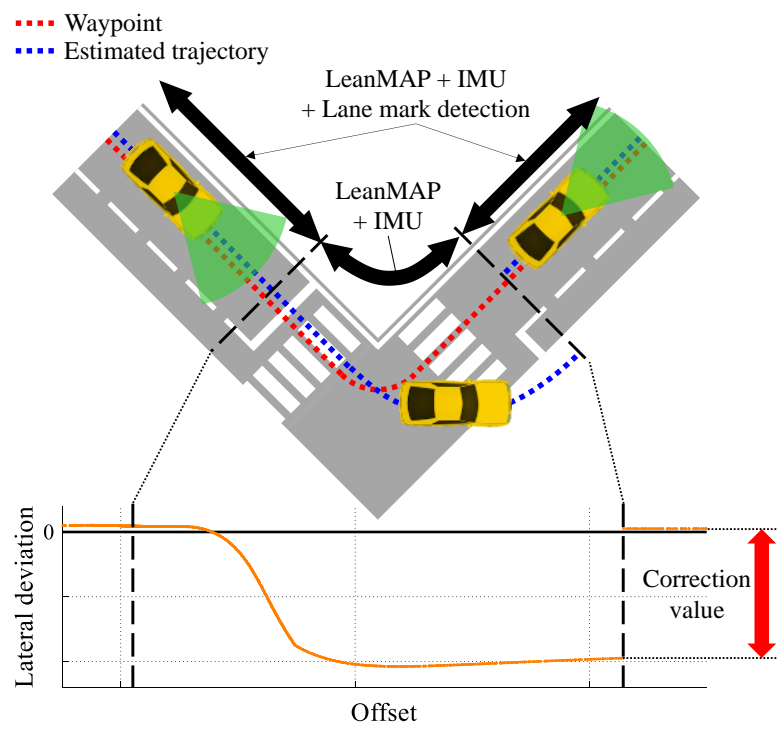

Fig. 20 Conceptual Schematic of the Correction Value at the Intersection Curve

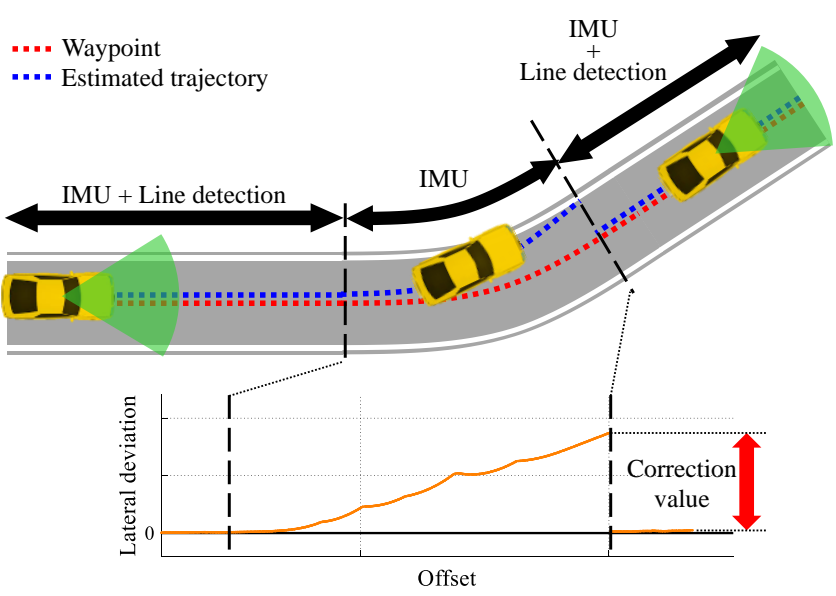

Fig. 21 Conceptual Schematic of the Correction Value at the Non-Intersection Curve

First, we evaluated the precision of the LeanMAP contents regarding the intersection curve. The evaluation area started from the point that was located approximately $5 \mathrm{~m}$ before the stop line. The extent of the area was approximately $50 \mathrm{~m}$. In this evaluation area, we ignored the lane mark detection. The correction value of the lateral deviation when the lane mark detection was reactivated represents the precision of the LeanMAP contents. Fig. 22 shows the evaluation result. The Float indicates the data before modification, whereas the Fix indicates the modified data. As shown in the figure, the correction value of the LeanMAP Fix is low compared to that of the LeanMAP Float. As discussed in our previous study ${ }^{(4)}$, the localization error of less than $0.5 \mathrm{~m}$ is one of the target values for automated driving at the intersection. Because the correction value discussed in this study was one of indices evaluating the localization error, this target value was accomplished, as evidenced by the results.

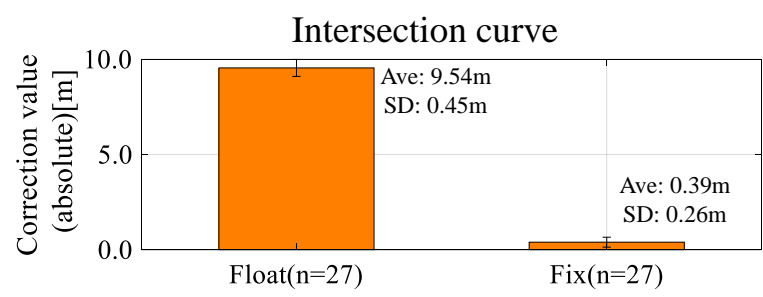

Fig. 22 Absolute Lateral Correction Value at the Intersection Curve

In a similar manner, we evaluated the precision of the LeanMAP contents regarding the non-intersection curve. The process was approximately same as that for the case of the intersection curve. The evaluation area started from the point that was located approximately $25 \mathrm{~m}$ before the first curve composition point. The extent of the area was approximately 120 $\mathrm{m}$. In this evaluation area, we ignored the lane mark detection. Fig. 23 shows the evaluation results. As in the case of the intersection curve, the modification of the LeanMAP contents decreases the correction value.

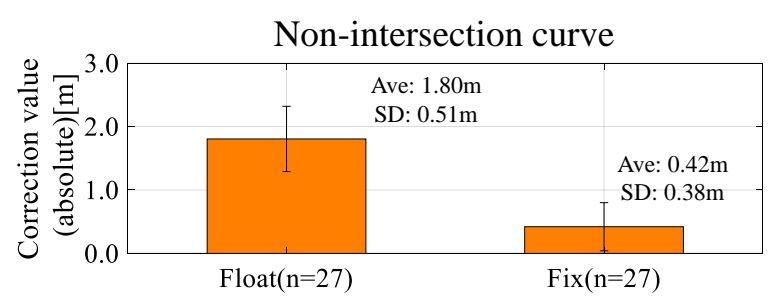

Fig. 23 Absolute Lateral Correction Value at the Non-Intersection Curve

\section{Conclusions}

To realize automated driving on non-highway roads, we proposed the creation of the digital map based on the LeanMAP framework, in which the digital map and the vehicular sensors act jointly in a complementary manner. In addition, we proposed a preliminary method for converting the digital map contents of car navigation systems to LeanMAP contents in order to enable the future preparation of nationwide digital map. Furthermore, we focused on the actual driving data, and we developed the modification system of the LeanMAP contents to compensate for the insufficient precision of the LeanMAP Float. To confirm the feasibility of the proposed system, we conducted an initial evaluation of the precision of the modified LeanMAP contents. Through the evaluation, we confirmed that our proposed system could update the LeanMAP Float to the LeanMAP Fix, and that their precision is sufficient for automated driving.

In this study, we focused on a test course that had a clear stop line in front of the intersection; thus, we could complete the modification process in a single link. However, stop lines and crosswalks may not be clear in narrow community roads; therefore, in our future work, we need to discuss an additional process for modification. In addition, as our test course did not include tight winding roads or steep slopes, we need to evaluate such characteristics roads in our future investigations. Moreover, we conducted both the modification process and the evaluation process using the same experimental vehicle; therefore, in our 
future study, it is important to further confirm the general applicability of the proposed method by using different experimental vehicles in the modification process and the evaluation process.

This paper is written based on a proceeding presented at JSAE 2017 Annual Congress (Autumn).

\section{Acknowledgement}

This research has been conducted as a part of the research project "Autonomous Driving System to Enhance Safe and Secured Traffic Society for Elderly Drivers" granted by Japan Science and Technology Agency (JST), S-Innovation (Strategic Promotion of Innovative Research and Development).

The data of test course were measured by the support of INCREMENT P CORPORATION. We deeply thank their contributions.

\section{References}

(1) Klaus Bengler et al.: Three Decades of Driver Assistance Systems, IEEE Intelligent Transportation Systems Magazine, Vol. 6, No. 4, pp. 6-22 (2014)

(2) Manabu Omae et al.: GPS-based Automatic Driving Control in Local Area with Course of Large Curvature and Parking Space, Vehicle System Dynamics 2004, Vol. 42, Nos. 1-2, pp. 59-73 (2004)

(3) Keisuke Yoneda et al.: Lidar Scan Feature for Localization with Highly Precise 3-D Map, 2014 IEEE Intelligent Vehicles Symposium Proceedings, pp. 1345-1350 (2014)

(4) Takuma Ito et al.: Novel Map Platform based on Primitive Elements of Traffic Environments for Automated Driving Technologies, International Journal of Automotive Engineering, Vol. 7, No. 4, pp. 143-151 (2016)

(5) Japan Digital Road Map Association: Data Model of Road Networks, Japan Digital Road Map Association, http://www.drm.jp/english/drm/database/expression.html

(Accessed July 13 2017) 